Mathematical and Statistical Economics, Vol. 2, 2020, no. 1, 17 - 27

HIKARI Ltd, www.m-hikari.com

https://doi.org/10.12988/mse.2020.986

\title{
Pricing Quanto Forward and European Options
}

\author{
Pascal Stiefenhofer \\ University of Exeter, Department of Economics, UK \\ and \\ University of Newcastle, Department of Economics, UK \\ Siddharth Kumar \\ University of Exeter, Department of Economics, UK \\ Andros Gregoriou \\ University of Brighton, Brighton Business School, UK
}

This article is distributed under the Creative Commons by-nc-nd Attribution License. Copyright (c) 2020 Hikari Ltd.

\begin{abstract}
In this paper, using Black-Scholes assumptions, we derive an analytical closed form solution for the pricing of a quanto forward and option contract. We use techniques of stochastic calculus and continuous time in order to establish a closed form solution for a quanto forward and option.
\end{abstract}

Keywords: Quanto Option Pricing, Quanto Forward Price, Closed Form Solution, Black-Scholes

\section{Introduction}

The pricing of quanto options using the Black-Scholes model (Black and Scholes, 1973) are extremely inaccurate in an empirical framework, due to the fat tails observed in financial returns (see among others, Mittnik et al, 2000 and Opschoor et al, 2018). Park et al (2013) price quanto options in a stochastic volatility framework, within the Black and Scholes model. However, the skewness of financial data makes their findings imprecise. Kim et al (2015) improve 
the Black and Scholes option pricing model for quanto options by introducing random time changes into normal tempered stable (NTS) distribution, which enables infinite jumps as well as capturing random varying time in stochastic volatility.

In this paper we present an analytical solution for the quanto forward and option prices, under Black-Scholes assumptions, which satisfies the general Feynman-Kac pricing partial differential equation (Janson and Tysk, 2006). In contrast with competing derivations assuming discrete time, the current research of developing a closed-form solution for quanto forwards and options allows the validation of the application of continuous time assumption and shows that errors can occur while adopting the former approach, especially when sampling frequencies are minuscule. Through this approach, extensions can be made to more complicated derivative securities, especially when they have dividend yield and intermittent income prior to the execution date. Also, numerical values can be very efficiently computed from the newly found analytical formula.

The next section introduces the notations and assumptions used in the pricing. The third section first prices the quanto forward contract. Based on the price of the quanto forward, we use the Black-Scholes pricing formula to price the European quanto option. Finally, we conclude by discussing possible shortcomings and how further research can improve the pricing, especially when parametric assumptions are changed.

\section{Assumptions and notation}

Consider a probability space $(\Omega, \mathcal{F}, P)$ where $\Omega$ is a set of outcomes, $\mathcal{F}$ a $\sigma$ field, and $P$ a probability measure and a time interval $\mathcal{T}=[0, T]$. For any $s, t \in \mathcal{T}$ with $s \leq t$ the family $\mathcal{F}_{t}$ is called a filtration. We assume an underlying share price $S$ follows a stochastic process. $S$ is adapted to the filtration $\mathcal{F}_{t}$, if $S_{t}$ can be determined by information available at time $t$. That is $S_{t}$ is $\mathcal{F}_{t^{-}}$ predictable for any $t \in \mathcal{T}$. The return process $X_{t}$ is a function of $S_{t}$, and $\langle X\rangle$ is the variation of $X$, which is known as the realized variance of the returns on $S . B_{t}$ is a Brownian motion, which is the limit, as $\triangle_{t} \rightarrow 0$, of a Gaussian discrete-time random walk.

$$
B_{t+\triangle_{t}}=B_{t}+\varepsilon_{t} \sqrt{\triangle_{t}}
$$

Where $\varepsilon_{t}$ are i.i.d standard normal distribution: $\varepsilon_{t} \sim N(0,1)$. An Itô process $X$ has the form:

$$
X_{t}=X_{0}+\int_{0}^{t} \mu_{s} d s+\int_{0}^{t} \sigma_{s} d B_{s}
$$


Where $X_{0}$ a constant, $\mu_{t}$ and $\sigma_{t}$ are adapted processes satisfying regularity conditions. The Itô process has the following properties:

$$
\begin{aligned}
d t \cdot d t & =0, \\
d B_{t} \cdot d t & =0, \\
d B_{t} \cdot d B_{t} & =d t .
\end{aligned}
$$

Consider a function $f(t, x)$ whose partial derivatives $\frac{\partial f}{\partial t}, \frac{\partial f}{\partial x}$ and $\frac{\partial f}{\partial x^{2}}$ are welldefined and continuous, and an Itô process $X_{t}$

$$
d X_{t}=\mu_{t} d t+\sigma_{t} d B_{t}
$$

This implies that $Y_{t}=f\left(t, X_{t}\right)$ is also an Itô process and

$$
\begin{aligned}
d Y_{t} & =\frac{\partial f}{\partial t} d t+\frac{\partial f}{\partial x} d X_{t}+\frac{1}{2} \frac{\partial^{2} f}{\partial x^{2}}\left(d X_{t}\right)^{2} \\
& =\left[\frac{\partial f}{\partial t}+\frac{\partial f}{\partial x} \mu_{t}+\frac{1}{2} \frac{\partial^{2} f}{\partial x^{2}} \sigma_{t}^{2}\right] d t+\frac{\partial f}{\partial x} \sigma_{t} d B_{t} .
\end{aligned}
$$

The second equality follows from the fact that $\left(d X_{t}\right)^{2}=\sigma_{t}^{2} d t$. The market is arbitrage-free if and only if there exists a risk-neutral measure $Q$.

According to Girsanov (1960), let $B$ be a Brownian motion and $P$ be the corresponding Wiener measure. Let $\eta_{t}$ be an adapted process satisfying Novikov's conditions. Then a process

$$
\xi_{t}=\exp \left(-\int_{0}^{t} \eta_{\tau} d B_{\tau}-\frac{1}{2} \int_{0}^{t} \eta_{\tau}^{2} d \tau\right)
$$

is a martingale and we can define a new measure $Q$ by

$$
Q(A)=\mathbb{E}^{P}\left(1_{A} \xi_{T}\right)
$$

equivalent to $P$. Equivalent means that measures $P$ and $Q$ agree which events have zero probability. Moreover, the process $B^{Q}$ defined by

$$
B_{t}^{Q}=B_{t}+\int_{0}^{t} \eta_{s} d s
$$

is a Brownian motion under $Q$. Then

$$
\mathbb{E}^{Q}\left[S_{T}\right]=\mathbb{E}^{P}\left[\xi_{T} S_{T}\right]
$$

Consider the discounted price process $S_{t} / S_{0, t}$. Assume there exists an equivalent martingale measure $Q$ such that for $T>t$, 


$$
\mathbb{E}_{t}^{Q}\left[\frac{S_{T}}{S_{0, T}}\right]=\frac{S_{t}}{S_{0, t}} .
$$

At this point in the analysis, we fix an arbitrary time horizon $T>0$ and introduce the following assumptions: (i) The real interest rate $r$ is constant. (ii) Markets are arbitrage-free. (iii) The underlying share price $S$ satisfies

$$
\frac{d S_{t}}{S_{t}}=r d t+\sigma_{t} d B_{t}, \quad S_{t}>0
$$

where $B_{t}$ is a Brownian motion. (iv)

$$
\int_{t}^{T} \sigma_{\tau}^{2} d \tau \text { is finite. }
$$

(v) $B_{t}$ and $B_{t}^{\sigma}$ are independent

$$
d B_{t} d B_{t}^{\sigma}=\rho d t
$$

From assumption (ii), we know that on a filtered probability space $(\Omega, \mathcal{F}, P)$, there exists an equivalent probability measure $Q$ such that $\forall \alpha, p \in \mathbb{R}$ and $\forall t \leq T$, a power contract paying at time $T, \alpha S_{T}^{p}$ has time- $t$ price equal to $\alpha \mathbb{E}_{t}^{\bar{Q}} S_{T}^{p}$, where $\mathbb{E}_{t}^{Q}$ denotes the $\mathcal{F}_{t^{-}}$conditional $Q$-expectation. We are now in the position to derive the pricing formulae for quanto forward contracts and quanto European options.

\section{Pricing}

Quanto Forward Contract: Assume that both the foreign asset price $S_{t}$ and the exchange rate $X_{t}$ follow a geometric Brownian motion:

$$
\begin{aligned}
\frac{d S_{t}}{S_{t}} & =\mu_{s} d t+\sigma_{s} d B_{t}^{s}, \\
\frac{d X_{t}}{X_{t}} & =\mu_{x} d t+\sigma_{x} d B_{t}^{x}, \\
d B_{t}^{s} d B_{t}^{x} & =\rho d t,
\end{aligned}
$$

where $S_{t}$ is the foreign asset price, and $X_{t}$ is the home price of one unit of the foreign currency. Let $r$ and $r_{f}$ be the domestic and the foreign interest rates 
respectively,

$$
\begin{aligned}
\frac{d S_{t}^{0}}{S_{t}^{0}} & =r d t \\
\frac{d S_{t}^{0, f}}{S_{t}^{0, f}} & =r_{f} d t
\end{aligned}
$$

Consider a quanto forward contract which pays $\bar{X} S_{T}-F$ at time $T$. Here $\bar{X}$ is a fixed exchange rate and $F$ is the quanto forward price in units of domestic currency. We derive $F$ that makes the market value of the quanto forward contract at time $t$ equal to zero. Let $\mathrm{V}$ be the value of the derivative security described. Given no arbitrage and the fundamental theorem of asset pricing we obtain $V_{T}=\bar{X} S_{T}-F$. We derive the price of a European quanto option that pays $\max \left(\bar{X} S_{T}-K, 0\right)$ at time $T$, risk-neutral valuation, and asset pricing equation. Under the risk-neutral measure $Q$, we obtain

$$
\begin{aligned}
\frac{V_{t}}{S_{t}^{0}} & =\mathbb{E}_{t}^{Q}\left[\frac{V_{T}}{S_{T}^{0}}\right], \\
V_{t} & =\frac{S_{t}^{0}}{S_{T}^{0}} \mathbb{E}_{t}^{Q}\left[V_{T}\right], \\
& =e^{-r(T-t)} \mathbb{E}_{t}^{Q}\left[V_{T}\right] .
\end{aligned}
$$

We require $F$ such that $V_{t}=0$, which means there is no money exchanged today. Hence

$$
\begin{aligned}
e^{-r(T-t)} \mathbb{E}_{t}^{Q}\left[V_{T}\right] & =0 \\
\mathbb{E}_{t}^{Q}\left[V_{T}\right] & =0 \\
\mathbb{E}_{t}^{Q}\left[\bar{X} S_{T}-F\right] & =0 \\
F & =\mathbb{E}_{t}^{Q}\left[\bar{X} S_{T}\right]
\end{aligned}
$$

In order to find this value, we need to study the dynamics of $S_{t}$ under riskneutral measure $Q$. Under $Q$, the asset price is a martingale as the return under $Q$ is equal to the risk free rate. Given that we have two Brownian motions $B_{t}^{s}$ and $B_{t}^{x}$; we assume that $\eta_{s}$ and $\eta_{x}$ are the prices of risks associated respectively. Now, by Girsanov's theorem we have

$$
\begin{aligned}
d B_{t}^{s, Q} & =d B_{t}^{s}+\eta_{s} d t \\
d B_{t}^{x, Q} & =d B_{t}^{x}+\eta_{x} d t
\end{aligned}
$$


We need to find $\eta_{x}$ and $\eta_{s}$ such that measure $Q$ is an equivalent Martingale measure, i.e. the discounted prices should be martingales. Let domestic and foreign risk price assets have prices $S_{t}^{0}$ and $S_{t}^{0, f}$, and

$$
\begin{aligned}
\frac{d S_{t}^{0}}{S_{t}^{0}} & =r d t \\
\frac{d S_{t}^{0, f}}{S_{t}^{0, f}} & =r_{f} d t .
\end{aligned}
$$

Due to the fact that these are risk-free assets, they do not have any diffusion (stochastic) terms. Steps (A)-(F) yield the requested results.

(A) From the domestic point of view, the foreign risky asset is an asset with risky price $Y_{t}=S_{t} X_{t}$. Applying Ito's lemma, we obtain a process for $d Y_{t}$. If $Y_{t}=f\left(t, S_{t}, X_{t}\right)$,

$$
\begin{aligned}
d Y_{t} & =f_{t} d t+f_{S_{t}} d S_{t}+f_{X_{t}} d X_{t}+\frac{1}{2}\left[f_{S_{t} S_{t}}\left(d S_{t}\right)^{2}+f_{X_{t} X_{t}}\left(d X_{t}\right)^{2}+2 f_{S_{t} X_{t}}\left(d S_{t}\right)\left(d X_{t}\right)\right] \\
& =0 d t+X_{t} S_{t}\left(\mu_{s} d t+\sigma_{s} d B_{t}^{s}\right)+S_{t} X_{t}\left(\mu_{x} d t+\sigma_{x} d B_{t}^{x}\right) \\
& +\frac{1}{2}\left[0\left(d S_{t}\right)^{2}+0\left(d X_{t}\right)^{2}\right. \\
& \left.+2\left(X_{t} S_{t}\left(\mu_{s} \mu_{x}(d t)^{2}\right)+\mu_{s} \sigma_{x} d B_{t}^{x} d t+\sigma_{s} d B_{t}^{s} d t+\sigma_{s} d B_{t}^{s} \mu_{x} d t+\sigma_{s} \sigma_{x} d B_{t}^{s} d B_{t}^{x}\right)\right] \\
& =X_{t} S_{t}\left(\mu_{s} d t+\sigma_{s} d B_{t}^{s}\right)+S_{t} X_{t}\left(\mu_{x} d t+\sigma_{x} d B_{t}^{x}\right)+X_{t} S_{t}\left(\sigma_{s} \sigma_{x} \rho d t\right) .
\end{aligned}
$$

Then we have

$$
\frac{d Y_{t}}{Y_{t}}=\left(\mu_{s}+\mu_{x}+\sigma_{x} \sigma_{x} \rho\right) d t+\sigma_{s} d B_{t}^{s}+\sigma_{x} d B_{t}^{x}
$$

(B) From the point of view of a domestic investor, the foreign risk free asset is a risky asset with price $Z_{t}=S_{t}^{0, f} X_{t}$. Applying Ito's lemma to get a process for $d Z_{t}$, where $Z_{t}=f\left(t, S_{t}^{0, f}, X_{t}\right)$. Thus

$$
\begin{aligned}
d Z_{t} & =f_{t} d t+f_{S} d S_{t}^{0, f}+f_{X} d X_{t}+\frac{1}{2}\left[f_{S S}\left(d S_{t}^{0, f}\right)^{2}+f_{X X}\left(d X_{t}\right)^{2}+2 f_{S X}\left(d S_{t}^{0, f}\right)\left(d X_{t}\right)\right] \\
& =0 d t+X_{t} S_{t}^{0, f} r_{f} d t+X_{t} S_{t}^{0, f}\left(\mu_{x} d t+\sigma_{x} d B_{t}^{x}\right) \\
& +\frac{1}{2}\left[0\left(d S_{t}^{0, f}\right)^{2}+0\left(d X_{t}\right)^{2}+2 S_{t}^{0, f} X_{t}\left(r_{f} \mu_{x}(d t)^{2}+r_{f} \sigma_{x} d B_{t}^{x} d t\right)\right] \\
& =X_{t} S_{t}^{0, f} r_{f} d t+X_{t} S_{t}^{0, f}\left(\mu_{x} d t+\sigma_{x} d B_{t}^{x}\right)+S_{t}^{0, f} x_{t}(0) \\
& =X_{t} S_{t}^{0, f} r_{f} d t+X_{t} S_{t}^{0, f}\left(\mu_{x} d t+\sigma_{x} d B_{t}^{x}\right),
\end{aligned}
$$


from which we obtain

$$
\frac{d Z_{t}}{Z_{t}}=\left(r_{f}+\mu_{x}\right) d t+\sigma_{x} d B_{t}^{x}
$$

Now, under the risk-neutral measure, the discounted prices of non-dividend paying assets must follow a martingale. Thus, we apply Ito's lemma to $d\left(\frac{Y_{t}}{S_{t}^{0}}\right)$ and $d\left(\frac{Z_{t}}{S_{t}^{0, f}}\right)$.

$$
\begin{aligned}
d\left(\frac{Y_{t}}{S_{t}^{0}}\right) & =0 d t+\frac{d Y_{t}}{S_{t}^{0}}+\left(\frac{-Y_{t}}{\left(S_{t}\right)^{2}}\right) d S_{t}^{0} \\
& +\frac{1}{2}\left[0\left(d Y_{t}\right)^{2}+\frac{2 Y_{t}}{\left(S_{t}^{0}\right)^{3}}\left(d S_{t}^{0}\right)^{2}+2 \frac{-1}{\left(S_{t}^{0}\right)^{2}} d S_{t}^{0} d Y_{t}\right] \\
& =\frac{Y_{t}}{S_{t}^{0}}\left[\left(\mu_{s}+\mu_{x}+\rho \sigma_{x} \sigma_{s}\right) d t+\sigma_{s} d B_{t}^{s}+\sigma_{x} d B_{t}^{x}\right]-\frac{Y_{t}}{S_{t}^{0}} r d t \\
& +\frac{1}{2}\left[\frac{2 Y_{t}}{\left(S_{t}^{0}\right)^{3}}\left(S_{t}^{0}\right)^{2} r^{2}(d t)^{2}-\frac{2 Y_{t} S_{t}^{0}}{\left(d S_{t}^{0}\right)^{2}}\left(r\left(\mu_{s}+\mu_{x}+\sigma_{x} \sigma_{s} \rho\right)(d t)^{2}+r \sigma_{x} d t d B_{t}^{x}\right)\right],
\end{aligned}
$$

from which we obtain

$$
d\left(\frac{Y_{t}}{S_{t}^{0}}\right) /\left(\frac{Y_{t}}{S_{t}^{0}}\right)=\left(\mu_{s}+\mu_{x}+\rho \sigma_{x} \sigma_{s}-r\right) d t+\sigma_{s} d B_{t}^{s}+\sigma_{x} d B_{t}^{x} .
$$

(D)

$$
\begin{aligned}
d\left(\frac{Z_{t}}{S_{t}^{0}}\right) & =0 d t+\frac{d Z_{t}}{S_{t}^{0}}+\left(\frac{-Z_{t}}{\left(S_{t}^{0}\right)^{2}}\right) d S_{t}^{0} \\
& +\frac{1}{2}\left[0\left(d Z_{t}\right)^{2}+\frac{2 Z_{t}}{\left(S_{t}^{0}\right)^{3}}\left(d S_{t}^{0}\right)^{2}+2 \frac{-1}{\left(S_{t}^{0}\right)^{2}} d S_{t}^{0} d Z_{t}\right] \\
& =\frac{Z_{t}}{S_{t}^{0}}\left[\left(r_{f}+\mu_{x}\right) d t+\sigma_{x} d B_{t}^{x}\right]-\frac{Z_{t}}{S_{t}^{0}} r d t \\
& +\frac{1}{2}\left[\frac{2 Z_{t}}{\left(S_{t}^{0}\right)^{3}}\left(S_{t}^{0}\right)^{2} r^{2}(d t)^{2}-\frac{2 z_{t} S_{t}^{0}}{\left(d S_{t}^{0}\right)^{2}}\left(r\left(r_{f}+\mu_{x}\right)(d t)^{2}+r \sigma_{x} d t d B_{t}^{x}\right)\right]
\end{aligned}
$$

From which we obtain

$$
d\left(\frac{Z_{t}}{S_{t}^{0}}\right) /\left(\frac{Z_{t}}{S_{t}^{0}}\right)=\left(r_{f}+\mu_{x}-r\right) d t+\sigma_{x} d B_{t}^{x}
$$


We use Girsanov's theorem to express (3) and (4) under the risk-neutral measure. Rewriting $\left(\frac{Y_{t}}{S_{t}^{0}}\right)$ and $\left(\frac{Z_{t}}{S_{t}^{0}}\right)$ under $Q$.

(E)

$d\left(\frac{Y_{t}}{S_{t}^{0}}\right) /\left(\frac{Y_{t}}{S_{t}^{0}}\right)=\left(\mu_{s}+\mu_{x}+\rho \sigma_{x} \sigma_{s}-r-\sigma_{s} \eta_{s}-\sigma_{x} \eta_{x}\right) d t+\sigma_{s} d B_{t}^{s, Q}+\sigma_{x} d B_{t}^{x, Q}$

$(\mathrm{F})$

$$
d\left(\frac{Z_{t}}{S_{t}^{0}}\right) /\left(\frac{Z_{t}}{S_{t}^{0}}\right)=\left(r_{f}+\mu_{x}-r-\sigma_{x} \eta_{x}\right) d t+\sigma_{x} d B_{t}^{x, Q}
$$

Thus, both processes (9) and (10) have zero drift under $Q$. We use this property in order to solve for $\eta_{s}$ and $\eta_{x}$. Therefore,

$$
\begin{aligned}
\sigma_{s} \eta_{s} & =\mu_{s}+\rho \sigma_{s}+\rho \sigma_{s} \sigma_{x}-r_{f} \\
\sigma_{x} \eta_{x} & =r_{f}+\mu_{x}-r
\end{aligned}
$$

yields

$$
\begin{aligned}
\frac{d S_{t}}{S_{t}} & =\left(\mu_{s}-\sigma_{s} \eta_{s}\right) d t+\sigma_{s} d B_{t}^{s, Q} \\
& =\left(r_{f}-\rho \sigma_{x} \sigma_{s}\right) d t+\sigma_{s} d B_{t}^{s, Q}
\end{aligned}
$$

Applying Itô's lemma to $\ln \left(S_{t}\right)$ yields

$$
\begin{aligned}
d \ln \left(S_{t}\right) & =\frac{d S_{t}}{S_{t}}-\frac{\left(d S_{t}\right)^{2}}{2 S_{t}^{2}} \\
& =\left(r_{f}-\rho \sigma_{x} \sigma_{s}\right) d t+\sigma_{s} d B_{t}^{s, Q} .
\end{aligned}
$$

Since $\left(d S_{t}\right)^{2}=S_{t}^{2} \sigma_{s}^{2} d t$,

$$
d \ln \left(S_{t}\right)=\left(r_{f}-\rho \sigma_{x} \sigma_{s}-\sigma_{s}^{2} / 2\right) d t+\sigma_{s} d B_{t}^{s, Q} .
$$

Integrating from $t$ to $T$ yields

$$
\begin{aligned}
\int_{t}^{T} d \ln \left(S_{\tau}\right) d \tau & =\int_{t}^{T}\left(r_{f}-\rho \sigma_{x} \sigma_{s}-\sigma_{s}^{2} / 2\right) d \tau+\int_{t}^{T} \sigma_{s} d B_{\tau}^{s, Q} d \tau . \\
\ln \left(S_{T}\right)-\ln \left(S_{t}\right) & =\left(r_{f}-\rho \sigma_{x} \sigma_{s}-\sigma_{s}^{2} / 2\right)(T-t)+\sigma_{s}\left(B_{T}^{s, Q}-B_{t}^{s, Q}\right) \\
S_{T} & =S_{t} \exp \left[\left(r_{f}-\rho \sigma_{x} \sigma_{s}-\sigma_{s}^{2} / 2\right)(T-t)+\sigma_{s}\left(B_{T}^{s, Q}-B_{t}^{s, Q}\right)\right] .
\end{aligned}
$$


We now conclude that

$$
F=\mathbb{E}_{t}^{Q}\left(\bar{X} S_{T}\right)=\bar{X} \mathbb{E}_{t}^{Q}\left(S_{T}\right)
$$

Using the property that $\mathbb{E} \exp x=\exp \left(\mathbb{E}(x)+\frac{1}{2} \operatorname{var}(x)\right)$ we derive

$$
\begin{aligned}
\mathbb{E}_{t}^{Q}\left(S_{T}\right) & =\mathbb{E}_{t}^{Q} S_{t} \exp \left[\left(r_{f}-\rho \sigma_{x} \sigma_{s}-\sigma_{s}^{2} / 2\right)(T-t)+\sigma_{s}\left(B_{T}^{s, Q}-B_{t}^{s, Q}\right)\right] \\
& =S_{t} \mathbb{E}_{t}^{Q} \exp \left[\left(r_{f}-\rho \sigma_{x} \sigma_{s}-\sigma_{s}^{2} / 2\right)(T-t)+\sigma_{s}\left(B_{T}^{s, Q}-B_{t}^{s, Q}\right)\right] \\
& =S_{t} \exp \left[\mathbb{E}_{t}^{Q}\left\{\left(r_{f}-\rho \sigma_{x} \sigma_{s}-\sigma_{s}^{2} / 2\right)(T-t)+\sigma_{s}\left(B_{T}^{s, Q}-B_{t}^{s, Q}\right)\right\}\right] \\
& +\frac{1}{2} \operatorname{var}\left\{\left(r_{f}-\rho \sigma_{x} \sigma_{s}-\sigma_{s}^{2} / 2\right)(T-t)+\sigma_{s}\left(B_{T}^{s, Q}-B_{t}^{s, Q}\right)\right\} \\
& =S_{t} \exp \left\{\left(r_{f}-\rho \sigma_{x} \sigma_{s}-\sigma_{s}^{2} / 2\right)(T-t)+\frac{1}{2} \sigma_{s}^{2}(T-t)\right\} \\
& =S_{t} \exp \left\{\left(r_{f}-\rho \sigma_{x} \sigma_{s}\right)(T-t)\right\} .
\end{aligned}
$$

It follows that

$$
F=\bar{X} S_{t} \exp \left[\left(r_{f}-\rho \sigma_{x} \sigma_{s}\right)(T-t)\right]
$$

as requested. Next, let's find the price of a European quanto option paying $\max \left(\bar{X} S_{T}-K, 0\right)$ at time $T$. Let this price be denoted by $P$. We apply the Black-Scholes pricing formula.

$$
\max \left(\bar{X} S_{T}-K, 0\right)=\bar{X} \max \left(S_{T}-\frac{K}{\bar{X}}, 0\right)
$$

Let $P^{\prime}$ be the price of an option that pays $\max \left(S_{T}-\frac{K}{\bar{X}}, 0\right)$ at maturity. Given no arbitrage and the fundamental theorem of asset pricing: $P=\bar{X} P^{\prime}$. Thus, we price $P^{\prime}$ first then multiply it by $\bar{X}$. This yields

$$
\begin{aligned}
\frac{d S_{t}}{S_{t}} & =\left(r_{f}-\rho \sigma_{x} \sigma_{s}\right) d t+\sigma_{s} d B_{t}^{s, Q} \\
\mathbb{E}_{t}^{Q}\left(\frac{d S_{t}}{S_{t}}\right) & =\left(r_{f}-\rho \sigma_{x} \sigma_{s}\right) d t \neq r d t .
\end{aligned}
$$

This is not an arbitrage because the foreign asset $S_{t}$ is denominated in foreign currency. At this point we cannot directly use the Black-Scholes formula. Let $q=r-r_{f}+\rho \sigma_{x} \sigma_{s}$, then

$$
\frac{d S_{t}}{S_{t}}=(r-q) d t+\sigma_{s} d B_{t}^{s, Q}
$$


This is now analogous to pricing an asset using the Black-Scholes formula with constant dividend yield $(q)$.

$$
\begin{aligned}
P^{\prime} & =\exp (-q(T-t)) S_{t} N\left(d_{1}\right)-\exp (-r(T-t)) \frac{K}{\bar{X}} N\left(d_{2}\right) \\
P & =\bar{X} P^{\prime}=\exp \left(\left(r_{f}-r-\rho \sigma_{x} \sigma_{s}\right)(T-t)\right) \bar{X} S_{t} N\left(d_{1}\right)-\exp (-r(T-t)) K N\left(d_{2}\right),
\end{aligned}
$$

where

$$
\begin{aligned}
& d_{1}=\ln \left(\frac{\bar{X} S_{t}}{K}+\left(r_{f}-\rho \sigma_{s} \sigma_{x}+\frac{\sigma_{s}^{2}}{2}\right)(T-t)\right) /\left(\sigma_{s} \sqrt{T-t}\right) \\
& d_{2}=d_{1}-\sigma_{s} \sqrt{T-t}
\end{aligned}
$$

\section{Conclusions}

Using Black-Scholes assumptions, this paper provides an analytical closed form solution for the pricing of a quanto forward and option contract. This is a desired contribution to the mathematical finance literature given that, most prior research obtains quanto option solutions using numerical techniques and Monte-Carlo simulations. Our solution provides very interesting avenues for further research. First, stochastic volatility could be incorporated into the pricing methodology (Heston, 1993), as both option and currency volatility is typically time-varying. More advanced derivatives can also be written on quanto, like quanto swaptions and quanto-forward floating-strike Asian options, for which higher parametric sophistication and processes for additional variables like interest rate would be required.

\section{References}

[1] Black, F. and Scholes, M., The pricing of options and corporate liabilities, Journal of Political Economy, 81 (3) (1973), 637-654.

https://doi.org/10.1086/260062

[2] Heston, S.L., A closed-form solution for options with stochastic volatility with applications to bond and currency options, The Review of Financial Studies, 6 (2) (1993), 327-343. https://doi.org/10.1093/rfs/6.2.327

[3] Girsanov, I. V., On transforming a certain class of stochastic processes by absolutely continuous substitution of measures, Theory of Probability and Its Applications, 5 (3) (1960), 285-301. https://doi.org/10.1137/1105027 
[4] Janson, S. and Tysk, J., Feynman-Kac formulas for Black-Scholes-type operators, Bulletin of the London Mathematical Society, 38 (2) (2006), 269-282. https://doi.org/10.1112/s0024609306018194

[5] Kim, Y.S., Lee, J., Mittnik, S. and Park, J., Quanto option pricing in the presence of fat tails and asymmetric dependence, Journal of Econometrics, 187 (2) (2015), 512-520. https://doi.org/10.1016/j.jeconom.2015.02.035

[6] Mittnik, S., Paolella, M.S. and Rachev, S.T., Diagnosing and treating the fat tails in financial returns data, Journal of Empirical Finance, 7 (3-4) (2000), 389-416. https://doi.org/10.1016/s0927-5398(00)00019-0

[7] Opschoor, A., Janus, P., Lucas, A. and Van Dijk, D., New HEAVY models for fat-tailed realized covariances and returns, Journal of Business $\&$ Economic Statistics, 36 (4) (2018), 643-657.

https://doi.org/10.1080/07350015.2016.1245622

[8] Park, J., Lee, Y. and Lee, J., Pricing of quanto option under the Hull and White stochastic volatility model, Communications of the Korean Mathematical Society, 28 (3) (2013), 615-633.

https://doi.org/10.4134/ckms.2013.28.3.615

Received: August 5, 2020; Published: August 31, 2020 